\title{
Photochemical Reaction of Polymers Having Arylketone and Tertiary Amine as Pendant Groups
}

\author{
Kyoji TsubakiYama, Masahiko Kuzuba, Kazuhide Yoshimura, \\ Masahide Yamamoto, ${ }^{*}$ and Yasunori NishiJima* \\ Research Institute for Material Science and Engineering, Faculty of Engineering, \\ Fukui University, Bunkyo, Fukui 910, Japan \\ * Department of Polymer Chemistry, Faculty of Engineering, Kyoto University, \\ Yoshida, Sakyo-ku, Kyoto 606, Japan
}

(Received September 7, 1990)

\begin{abstract}
Binary $\left(M_{1} / M_{2}\right)$ and ternary $\left(M_{1} / M_{2} / M_{3}\right)$ copolymer having aryl ketone and tertiary amine as pendant groups were prepared by radical polymerization, where $\mathbf{M}_{1}$ is 4-vinylbenzophenone (VBP) or 4-(2-naphthoyl)styrene (NSt), $\mathrm{M}_{2}$ is $2-N, N$-dimethylaminoethyl methacrylate (DMAEMA) or $4-N, N$-dimethylaminostyrene (DMASt), and $\mathrm{M}_{3}$ is methyl methacrylate. The polymer film was irradiated with a high-pressure mercury lamp $(\lambda>300 \mathrm{~nm})$, and photocrosslinking was followed by measuring the gel fraction. Crosslinking of the $\operatorname{VBP}\left(n, \pi^{*}\right)$-polymer was greatly accelerated by the amines, whereas that of the $\operatorname{NSt}\left(\pi, \pi^{*}\right)$-polymer could be induced only in their presence. The effects of the aliphatic amine (DMAEMA) were larger than those of the aromatic amine (DMASt). Absorption and emission spectra showed that the arylketones interact more strongly with aromatic amine than aliphatic amine. Laser photolysis experiments confirmed that the ketyl radicals were formed in the copolymers.

KEY WORDS Photocrosslinking / Ketone-Amine Copolymer / CT-

Interaction / Phosphorescence / Laser Photolysis / Ketyl Radical /

Poly(4-vinylbenzophenone) / Poly[4-(2-naphthoyl)styrene] /
\end{abstract}

The photoreaction of arylketones with amines has been extensively studied from the standpoint of organic photochemistry ${ }^{1,2}$ and used for the efficient photoinitiation of polymerization and UV curing systems. ${ }^{3-5}$ It has been proposed that an electron is transferred from the amine to the triplet state of the ketone, forming a charge-transfer (CT) complex; this complex then collapses by proton transfer, generating the initiating radicals. ${ }^{6,7}$ This photoreaction process has the following special features: a higher reaction rate than that of "normal" hydrogen-abstraction by $n, \pi^{*}$ arylketones 1 ; smooth progress of reaction of $\pi, \pi^{*}$-arylketones with amines ${ }^{1,8}$; insensitivity to oxygen inhibitation. ${ }^{9,10}$ On the other hand, it is well-known that poly(vinylbenzophenone) undergoes photocrosslinking through hydro- gen-abstraction on the polymer chain. ${ }^{11,12}$ Recently, Smets et al. ${ }^{13}$ investigated photocrosslinking and electron donor-acceptor interactions in copolymers of 4-vinylbenzophenone (VBP) with 4- $N, N$-dimethylaminostyrene (DMASt) and showed the merits of the incorporation of benzophenone and aromatic tertiary amines into a polymer chain.

In the present study, we prepared binary and ternary copolymers of arylketonic vinyl monomers such as VBP and 4-(2-naphthoyl)styrene (NSt) with various monomers containing aliphatic or aromatic tertiary amines (Figure 1), and investigated the effects of a combination of pendant ketones $\left(n, \pi^{*}\right.$ or $\left.\pi, \pi^{*}\right)$ with tertiary amino groups (aliphatic or aromatic) on the photocrosslinking reaction and the spectroscopic properties of the polymers. 


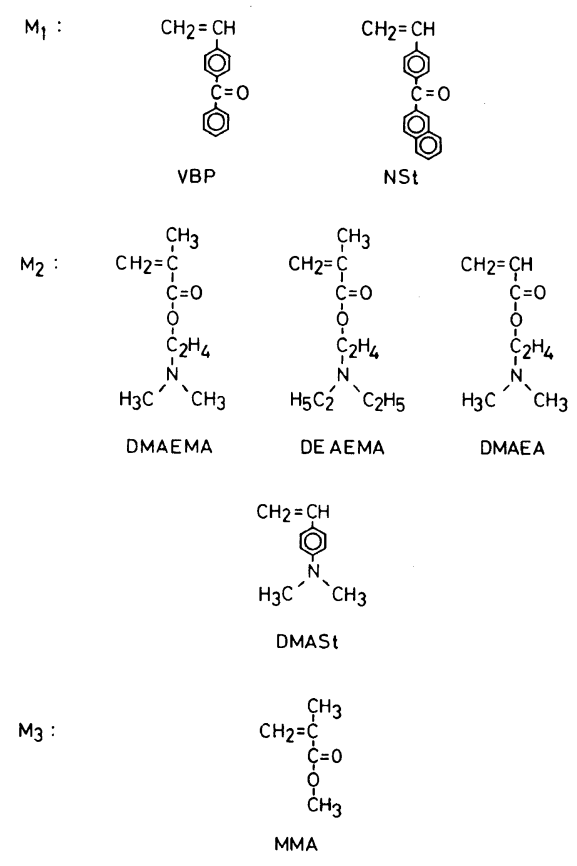

Figure 1. Structural formulas of monomers.

\section{EXPERIMENTAL}

\section{Materials}

The NSt monomer was synthesized from 4-chlorostyrene and 2-naphthonitrile in the same manner as VBP, ${ }^{14}$ and purified by column chromatography on silica gel and recrystallized from $n$-hexane: colorless crystals; mp 81.8$82.9^{\circ} \mathrm{C} .{ }^{1} \mathrm{H} \mathrm{NMR}\left(90 \mathrm{MHz}, \mathrm{CDCl}_{3}\right) \delta 5.41(1 \mathrm{H}$, dd, $J=11$ and $1 \mathrm{~Hz}), 5.89(1 \mathrm{H}$, dd, $J=18$ and $1 \mathrm{~Hz}), 6.81(1 \mathrm{H}, \mathrm{dd}, J=18$ and $11 \mathrm{~Hz})$, 7.47-7.70 (4H, m), 7.78-7.96 (6H, m), 8.25 ppm $\left(1 \mathrm{H}\right.$, br. s). Anal. Calcd for $\mathrm{C}_{19} \mathrm{H}_{14} \mathrm{O}$ : C, $88.34 \%$; H, 5.46\%. Found: C, $88.23 \%$; H, $5.61 \%$.

DMASt was prepared from 4- $N, N$-dimethylaminobenzaldehyde $^{15}$ and distilled under vacuum prior to use. Purchased DMAEMA, 2- $N, N$-diethylaminoethyl methacrylate (DEAEMA), 2- $N, N$-dimethylaminoethyl acrylate (DMAEA), and methyl methacrylate (MMA) were purified by distillation. 2Methyltetrahydrofuran (MTHF) was purified by passing through an alumina column, distillation over calcium hydride, and final vacuum distillation. Other solvents were purified by usual distillation.

\section{Copolymers}

Copolymers $\left(\mathbf{M}_{1} / \mathbf{M}_{2}\right)$ were obtained by the polymerization of $M_{1}$ with $M_{2}$ monomers in benzene solution containing AIBN at $60^{\circ} \mathrm{C}\left(\mathrm{MW}=8-12 \times 10^{4}\right)$. Terpolymers $\left(\mathrm{M}_{1} / \mathrm{M}_{2} / \mathrm{M}_{3}\right)$ were prepared by bulk polymerization of appropriate monomer mixtures in a similar manner $\left(\mathrm{MW}=12-50 \times 10^{4}\right)$. Here, $\mathbf{M}_{1}$ is arylketonic monomer, $\mathbf{M}_{2}$ is amine monomer, $\mathbf{M}_{3}$ is MMA, as shown in Figure 1. Polymers obtained were precipitated in $n$ hexane, and further purified by reprecipitation and dried at $40^{\circ} \mathrm{C}$ under vacuum. The copolymers containing the aliphatic amine are colorless, whereas the copolymer of the aromatic amine has a pale orange color. Compositions of the polymers were determined by elemental analysis. From the results of binary copolymerization, the reactivity ratios were roughly estimated by the Finemann-Ross method: $r_{1}=0.8$ and $r_{2}=0.2$ for $\operatorname{VBP}\left(M_{1}\right) /$ $\operatorname{DMASt}\left(\mathrm{M}_{2}\right), \quad r_{1}=0.6$ and $r_{2}=0.1$ for $\operatorname{NSt}\left(\mathrm{M}_{1}\right) / \mathrm{DMASt}\left(\mathrm{M}_{2}\right), r_{1}=3.0$ and $r_{2}=0.4$ for $\operatorname{VBP}\left(\mathrm{M}_{1}\right) / \operatorname{DMAEMA}\left(\mathrm{M}_{2}\right), r_{1}=2.8$ and $r_{2}=$ 0.6 for $\operatorname{NSt}\left(M_{1}\right) / \operatorname{DMAEMA}\left(\mathrm{M}_{2}\right)$.

\section{Photocrosslinking}

A transparent polymer film $(40-100 \mu \mathrm{m}$ thickness) coated on a glass plate was irradiated with a light of wavelength longer than $300 \mathrm{~nm}$ from a $100 \mathrm{~W}$ high-pressure mercury lamp (Ushio UM-100B) in air at room temperature. Photocrosslinking was followed by measuring the gel fraction (wt\%) after extraction of the soluble part with dichloromethane using a Soxhlet extractor.

\section{Spectroscopic Measurements}

Absorption spectra were obtained using a JASCO UV-430 spectrophotometer. Phosphorescence spectra were measured with a Hitachi Model-850 spectrofluorophotometer 
fitted with a phosphorescence attachment. Transient absorption spectra were recorded with a nanosecond laser photolysis apparatus $\left(347 \mathrm{~nm}\right.$ excitation, $14 \mathrm{~ns}$ pulse duration). ${ }^{16}$

\section{RESULTS AND DISCUSSION}

\section{Absorption Spectrum}

The UV spectrum of poly(VBP) measured in chloroform showed two absorption bands at $261 \mathrm{~nm}\left(\varepsilon=2.1 \times 10^{4}\right)$ and $336 \mathrm{~nm}(\varepsilon=2.2 \times$ $\left.10^{2}\right)$ and that of poly(NSt) also showed two maxima at $258 \mathrm{~nm}\left(\varepsilon=3.7 \times 10^{4}\right)$ and $336 \mathrm{~nm}$ $\left(\varepsilon=2.3 \times 10^{3}\right)$. Poly(DMAEMA) exhibited a weak absorption band at $243 \mathrm{~nm}\left(\varepsilon=2 \times 10^{2}\right)$
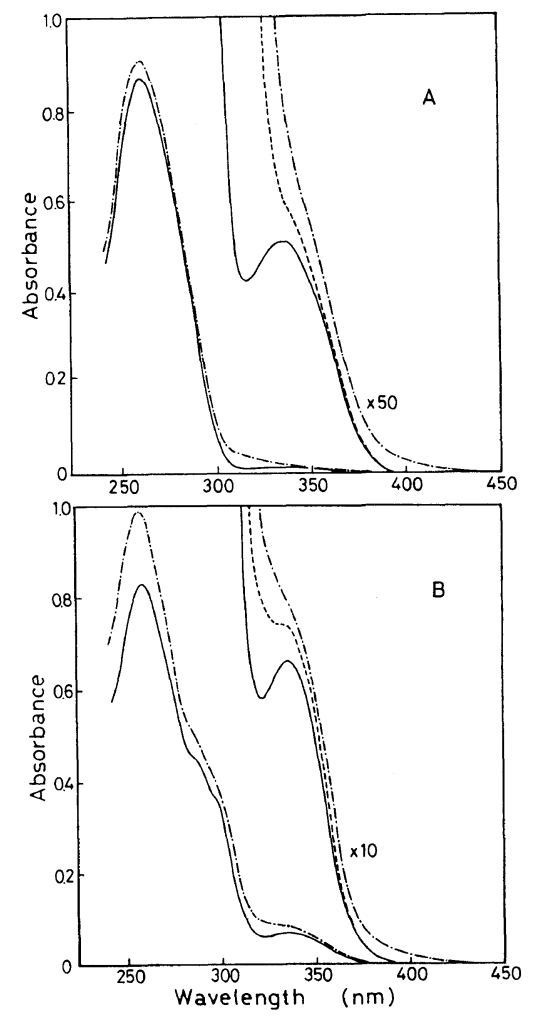

Figure 2. UV absorption spectra of copolymers in chloroform. A: (-) poly[VBP(92)/DMAEMA(8)]; (一.-) poly[VBP(79)/DMASt(21)]. [B]: (一) poly[NSt(59)/DMAEMA(41)]; (-.-) poly[NSt(61)/ DMASt(39)]; (---) calculated spectra obtained by summation of spectra of poly(VBP) or poly(NSt) and poly(DMASt). and poly(DMASt) had two bands at $254 \mathrm{~nm}$ $\left(\varepsilon=1.2 \times 10^{4}\right)$ and $310 \mathrm{~nm}\left(\varepsilon=2 \times 10^{3}\right)$.

Figure 2 shows the UV absorption spectra of four copolymers $\left(\mathrm{M}_{1} / \mathbf{M}_{2}\right)$ in chloroform. The spectra of copolymers (VBP/DMAEMA) and (NSt/DMAEMA) were found to be the same as those of poly(VBP) and poly(NSt), respectively. This indicates that no electronic interaction between the arylketone and alkylamine residues of polymers exists in the ground state. ${ }^{2}$ On the other hand, the copolymers (VBP/DMASt) and (NSt/DMASt) gave larger absorbance at $320-430 \mathrm{~nm}$ than the sum of absorbances for the corresponding two homopolymers, as shown in the figure. This increase in absorption is attributable to an electron donor-acceptor interaction between the arylketone and the arylamine residues of copolymers. ${ }^{13}$

\section{Photocrosslinking}

In order to compare the effects of alkylamine on the photocrosslinking with that of arylamine, the films of copolymers $\left(\mathbf{M}_{1} / \mathbf{M}_{2}\right)$ of similar compositions were first irradiated and the fraction of the gels produced was measured. The results are summarized in Table I. It has been found that DMAEMA is more effective for crosslinking than DMASt in both VBP-and NSt-copolymers. This suggests that arylamine is less favorable for photocrosslinking than

Table I. Photogelation of copolymers $\left(\mathbf{M}_{1} / \mathbf{M}_{2}\right)^{\mathbf{a}}$

\begin{tabular}{|c|c|c|c|}
\hline \multirow{2}{*}{ Run } & \multirow{2}{*}{$\begin{array}{c}\mathbf{M}_{1}(\%) / \mathbf{M}_{2}(\%) \\
\text { in copolymer }\end{array}$} & \multirow{2}{*}{$\frac{\text { Time }}{\min }$} & \multirow{2}{*}{$\frac{\text { Gel fraction }}{(\%)}$} \\
\hline & & & \\
\hline 1 & VBP(61)/DMASt(39) & 2 & 4 \\
\hline 2 & VBP(61)/DMASt(39) & 10 & 27 \\
\hline 3 & VBP(57)/DMAEMA(43) & 2 & 36 \\
\hline 4 & VBP(57)/DMAEMA(43) & 10 & 63 \\
\hline 5 & NSt(61)/DMASt(39) & 2 & 3 \\
\hline 6 & NSt(61)/DMASt(39) & 10 & 13 \\
\hline 7 & NSt(59)/DMAEMA(41) & 2 & 8 \\
\hline 8 & NSt(59)/DMAEMA(41) & 10 & 22 \\
\hline
\end{tabular}

a Films were irradiated $(\lambda>300 \mathrm{~nm})$ in air at room temperature. 

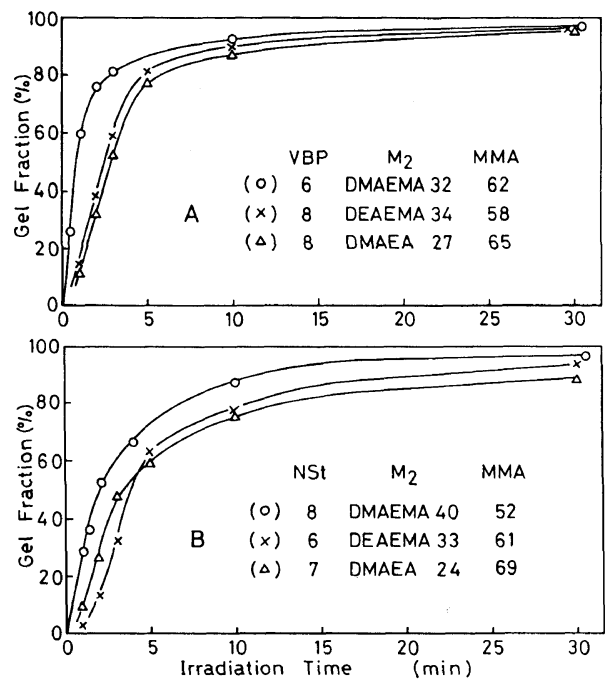

Figure 3. Effects of different $\mathbf{M}_{2}$ monomers on photocrosslinking of $\mathrm{A}$ terpolymer (VBP/M $\left./ \mathrm{M}_{2} / \mathrm{MMA}\right)$ and $\mathrm{B}$ terpolymer (NSt/M $\left./ \mathrm{M}_{2} / \mathrm{MMA}\right)$.
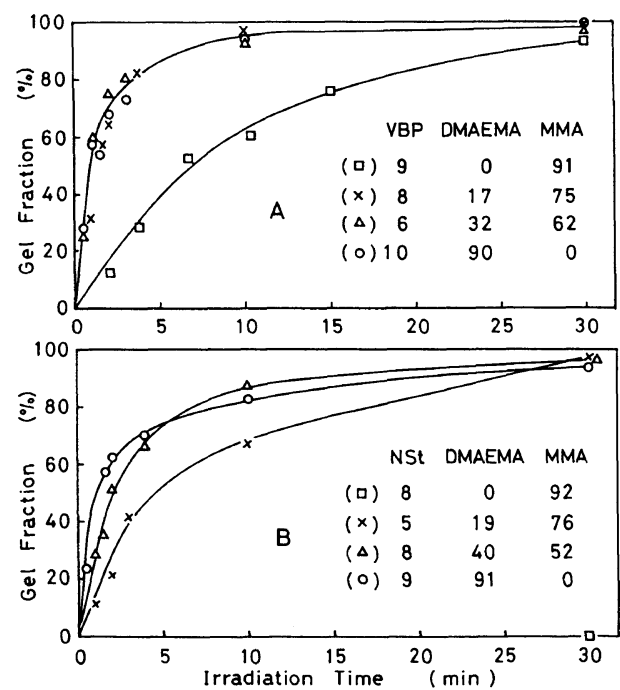

Figure 4. Effects of DMAEMA( $\left.\mathrm{M}_{2}\right)$ content on photocrosslinking of A terpolymer (VBP/DMAEMA/MMA) and B terpolymer (NSt/DMAEMA/MMA).

alkylamine.

The photocrosslinking reactions of terpolymers $\left(M_{1} / M_{2} / M_{3}\right)$ containing various aliphatic amine monomers as $\mathbf{M}_{2}$ were next examined. The content of the arylketone monomer unit in the terpolymer, which is a light-absorbing

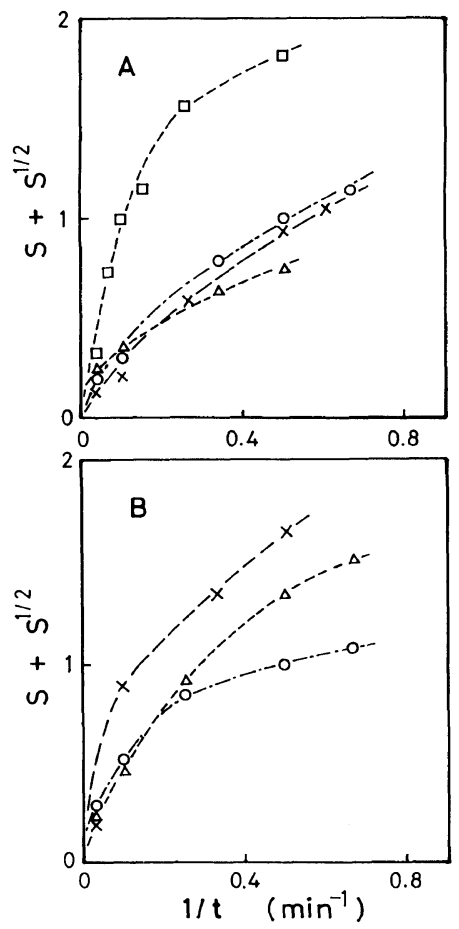

Figure 5. Charlesby-Pinner plots for A terpolymers (VBP/DMAEMA/MMA) and B terpolymers (NSt/ DMAEMA/MMA). Values of $S$ are based on the results of Figure 4.

chromophore, was held constant at $6-8 \%$. As can be seen from Figure 3, the accelerating effect of three amine monomers on the crosslinking increased in the order of DMAEMA $>$ DEAEMA $\simeq$ DMAEA in both VBP- and NSt-terpolymers.

Figure 4 shows the effects of the content of DMAEMA $\left(\mathrm{M}_{2}\right)$, which is the most effective monomer among amine monomers used, on the photocrosslinking of the terpolymers with similar $\mathrm{M}_{1}$ contents. In the case of terpolymer (VBP/DMAEMA/MMA) (Figure 4A), crosslinking was enhanced significantly by the coexistence of the amine monomers, though it occurred slowly in the absence of the amine. The amine content of $20-30 \%$ seems to be enough for the effective acceleration of photocrosslinking. In the case of terpolymer (NSt/DMAEMA/MMA) (Figure 4B), on the 
other hand, the photocrosslinking took place only when the amine monomer coexisted, and the reaction rate increased with increasing content of the amine monomer.

Photocrosslinking reactions were analyzed by the Charlesby-Pinner plots according to the equation $^{17}$ :

$$
S+S^{1 / 2}=\beta / \alpha+1 / \alpha P_{n} D
$$

where the sol-fraction $(S)$ was based on the results of Figure 4 and the absorbed light energy $(D)$ was taken to be proportional to the irradiation time $(t)$. As can be seen from Figure 5 , the plots are curved for both VBP- and NSt-terpolymers; this may be attributable to the heterogeneous reactions in solid state. From these results, however, the ratio of degradation to crosslinking $(\beta / \alpha)$ can be estimated as almost zero; that is, photocrosslinking is scarcely accompanied by the main chain scission..$^{9,10}$

\section{Change of Absorption Spectrum}

Figure 6 shows changes of absorption spectra of the copolymer (VBP/DMAEMA) films with irradiation. It was found that absorption around $260 \mathrm{~nm}$ due to the benzophenone group rapidly decreased with increasing irradiation time, whereas the absorbance in the region above $300 \mathrm{~nm}$ increased. These

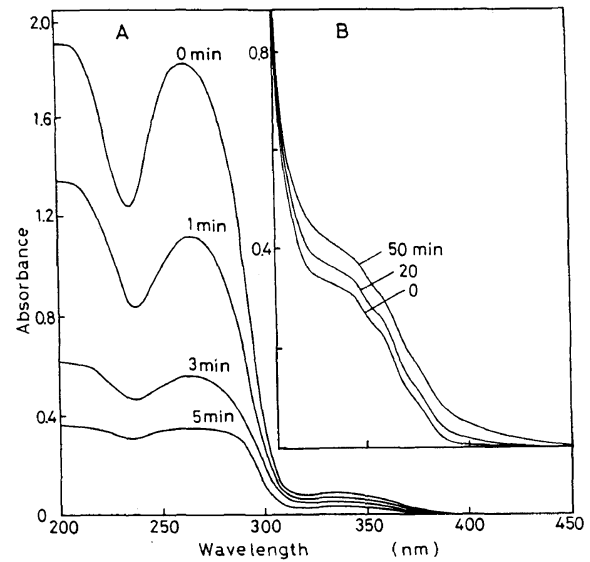

Figure 6. Change of absorption spectrum of polymer film with irradiation. A, copolymer [VBP(92)/DMAEMA(8)]; B, copolymer [VBP(10)/DMAEMA(90)]. changes in the absorption spectrum indicate that the phenone structure in the polymer was broken by photoreduction, resulting in the formation of some coupling products of the ketyl radicals. ${ }^{11}$ The copolymer in solution also exhibited similar spectral changes with irradiation, as shown in Figure 7 . In this case, the increase of absorbance in the longer wavelength region is attributable to the formation of ortho- and para-coupling products of the isomerized ketyl radicals. ${ }^{18}$

For the copolymer (NSt/DMAEMA), as shown in Figure 8, decreasing the absorption around $330 \mathrm{~nm}$ due to the naphthophenone group and increasing the absorbance in the region above $350 \mathrm{~nm}$ were also observed; this

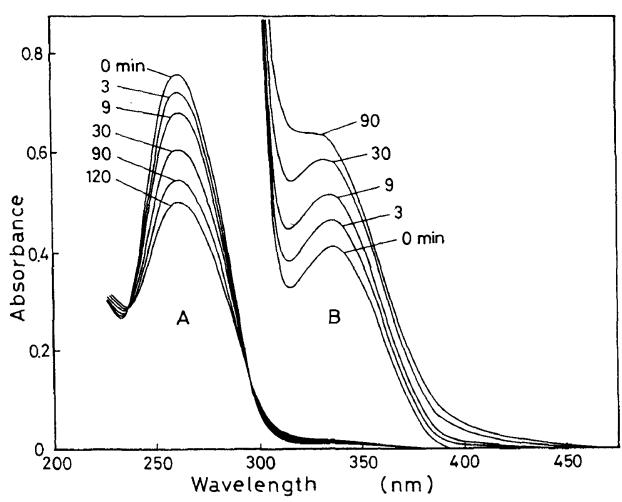

Figure 7. Change of absorption spectrum with irradiation for the copolymer $[\operatorname{VBP}(69) / \operatorname{DMAEMA}(31)]$ in $\mathrm{CH}_{2} \mathrm{Cl}_{2}$ solution. $\mathrm{A},[\mathrm{VBP}]=1 \times 10^{-4} \mathrm{M} ; \mathrm{B},[\mathrm{VBP}]=4 \times$ $10^{-3} \mathrm{M}$.

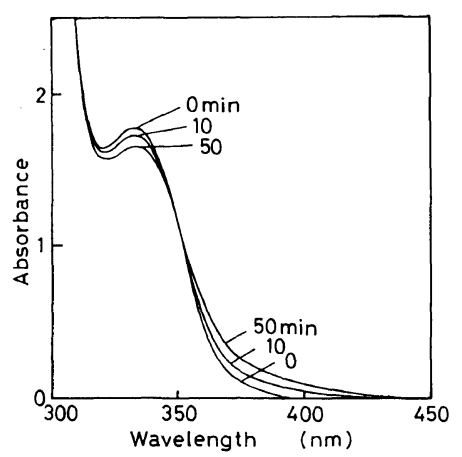

Figure 8. Change of absorption spectrum of copolymer [NSt(6)/DMAEMA(94)] film with irradiation. 
suggests that the phenone was photo-reduced and ketyl radicals were formed. ${ }^{8}$

\section{Emission Spectrum}

The phosphorescence spectra for four copolymers $\left(\mathbf{M}_{1} / \mathbf{M}_{2}\right)$ of similar compositions were measured in MTHF solid solution at a low temperature. Figure 9A shows the spectra of VBP-copolymers. The copolymer (VBP/ DMAEMA) gave the same spectrum as that of poly-(VBP). ${ }^{19}$ On the other hand, the copolymer (VBP/DMASt) exhibited a weak and broad emission around $480 \mathrm{~nm}$; this is ascribed to a triplet exciplex between the benzophenone and dimethylaminobenzene groups in the .polymer. ${ }^{2,13}$ These results indicate that the excited triplet benzophenone $\left(n, \pi^{*}\right)$ interacts more strongly with the aromatic amine than the alphatic amine in the polymer. The spectra of NSt-copolymers are shown in Figure 9B. The copolymers (NSt/
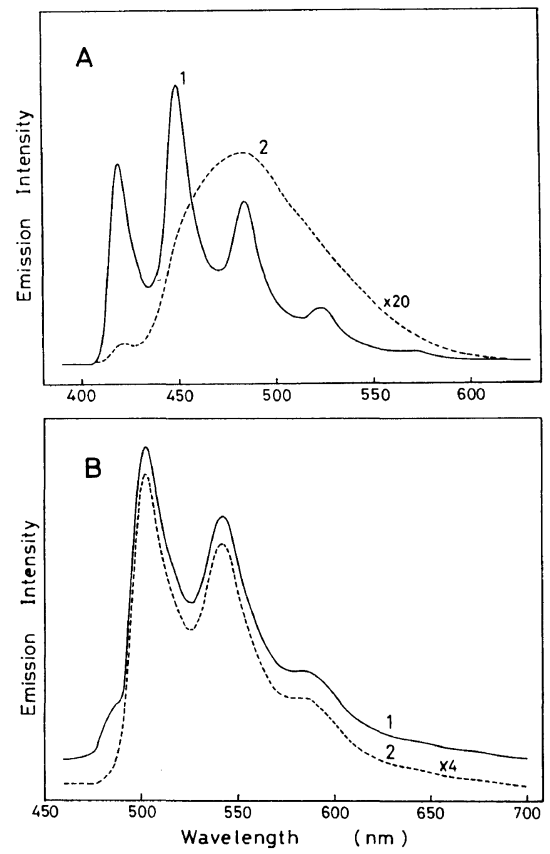

Figure 9. Phosphorescene spectra of copolymers in MTHF solid solution at $77 \mathrm{~K}\left(\lambda_{\text {ex }}=336 \mathrm{~nm}\right)$. A: (1) copolymer [VBP(57)/DMAEMA(43)]; (2) copolymer [VBP(61)/DMASt(39)]. B: (1) copolymer [NSt(59)/ DMAEMA(41)]; (2) copolymer [NSt(61)/DMASt(39)].
DMAEMA) and (NSt/DMASt) showed almost the same spectra as that of poly(NSt). ${ }^{19,20}$ However, the emission intensity of the former was higher than that of the latter by a factor of about 4; this indicates that the excited naphthophenone $\left(\pi, \pi^{*}\right)$ also interacts more strongly with the aromatic amine than the aliphatic amine.

\section{Laser Photolysis}

Transient absorption spectra for copolymer (NSt/DMAEMA) in MTHF and for 4-ethylphenyl-2-naphthyl ketone (EPNK)/triethylamine (TEA) in benzonitrile were measured at room temperature with a $347 \mathrm{~nm}$ laser flash. As shown in Figure 10A, the spectrum of the EPNK/TEA system observed immediately after excitation has two maxima at $c a .430$ and $650 \mathrm{~nm}$; this spectrum can be attributed to the triplet state. ${ }^{21,22}$ The spectrum observed at $7 \mu \mathrm{s}$
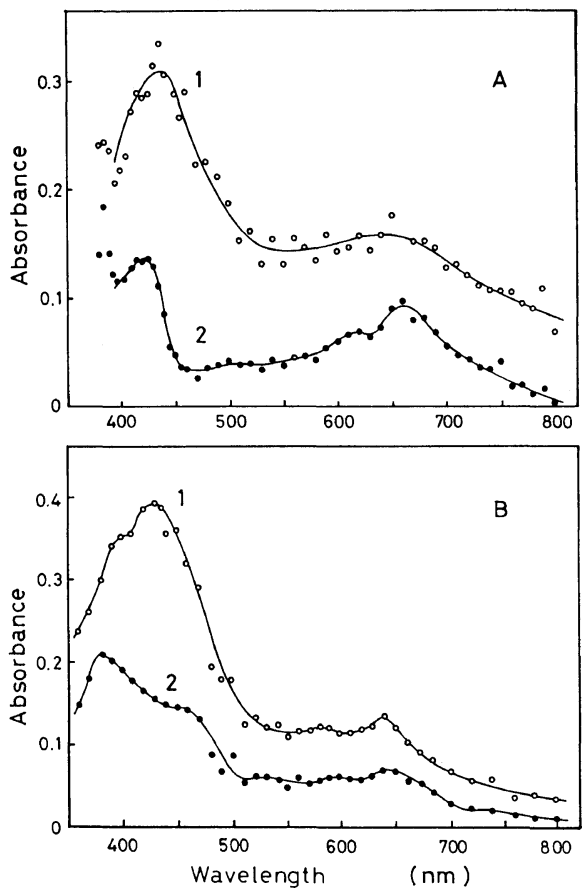

Figure 10. Transient absorption spectra measured by nanosecond laser photolysis. A: EPNK/TEA in benzonitrile observed (1) immediately after and (2) $7 \mu$ s after a laser pulse. B: copolymer [NSt(68)/DMAEMA(32)] in MTHF observed (1) immediately after and (2) $0.2 \mu$ s after. 
after excitation also has two maxima around 420 and $660 \mathrm{~nm}$, attributable primarily to the ketyl radical. The copolymer (NSt/DMAEMA) exhibited almost the same change in the transient spectrum, as shown in Figure 10B. It can be seen from this figure that the spectrum of the triplet naphthophenone group in the polymer $\left(\lambda_{\max } \simeq 430\right.$ and $\left.640 \mathrm{~nm}\right)$ changed to that of the ketyl radical $\left(\lambda_{\max } \simeq 380\right.$ and $640 \mathrm{~nm})$.

\section{Reaction Mechanism}

As described above, photocrosslinking of the polymer bearing benzophenone $\left(n, \pi^{*}\right)$ does not always require an amine to coexists, but the addition of a small amount of tertiary amine accelerates the reaction to a great extent. Crosslinking of the polymer having naphthophenone $\left(\pi, \pi^{*}\right)$ always needs an amine and its rate increases with increasing amine content. The accelerating effect of the aliphatic tertiary amine is larger than that of the aromatic amine.

The photocrosslinking of polymers containing pendant arylketone $(\mathbf{K})$ and tertiary amine (A) is considered to be initiated by ketyl and $\alpha$-aminoalkyl radicals on polymers produced through CT-interactions between $\mathbf{K}$ and $\mathbf{A},{ }^{13}$ as shown in Scheme 1. In this photoreaction, electron-transfer and subsequent proton-transfer processes are involved. ${ }^{1,23}$ The results of absorption and emission studies suggest that the photoreaction occurs via the following two pathways. In path (a), the excited triplet ketone reacts with the ground state amine through CT-interaction; this is the case of aliphatic

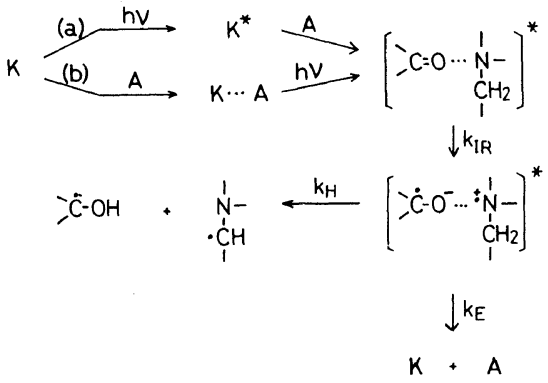

Scheme 1. amine copolymers. In path (b), the reaction is induced by photoexcitation of the ground state CT-complex. In the case of aromatic amine copolymers, both path (a) and (b) may be concernred in the photoreaction.

The results of spectroscopic analyses indicate that arylketone tends to interact more strongly with the aromatic amine than the aliphatic amine in both the ground and excited states. As mentioned above, however, the aliphatic tertiary amine is more effective for photocrosslinking than the aromatic amine. It seems therefore that the proton transfer process $\left(k_{\mathrm{H}}\right)$ rather than the electron transfer process $\left(k_{\mathrm{IR}}\right)$ plays an important role in this photocrosslinking reaction. The electron-transferred complex may be quenched either by proton-transfer reaction, generating ketyl and $\alpha$-aminoalkyl radicals, or by back-electron-transfer reaction, resulting in ground-state $\mathbf{K}$ and $\mathbf{A}^{1,23}$ Consequently, it is thought that the yield of initiating radicals, the efficiency of photocrosslinking, depends on the ratio of the rates $\left(k_{\mathrm{H}}, k_{\mathrm{E}}\right)$ of two competitive reactions.

\section{REFERENCES}

1. S. G. Cohen, A. Parola, and G. H. Parsons, Jr., Chem. Rev., 73, 141 (1973); S. Inbar, H. Linschitz, and S. G. Cohen, J. Am. Chem. Soc., 103, 1048 (1981).

2. S. Arimitsu, H. Masuhara, N. Mataga, and $\mathbf{H}$. Tsubomura, J. Phys. Chem., 79, 1255 (1975).

3. J. Guillet, "Polymer Photophysics and Photochemistry," Cambridge University Press, Cambridge, 1985, Chapter 10.

4. J. Hutchinson and A. Ledwith, Adv. Polym. Sci., 14, 49 (1974); A. Ledwith, J. A. Bosley, and M. D. Purbrick, J. Oil Col. Chem. Assoc., 61, 95 (1978).

5. J. P. Fouassier, J. Chim. Phys., 80, 339 (1983); G. Berner, R. Kirchmayr, and G. Rist, J. Oil Col. Chem. Assoc., 61, 105 (1978).

6. M. R. Sandner, C. L. Osborn, and D. J. Trecker, J. Polym. Sci., A-1, 10, 3173 (1972).

7. P. Ghosh and R. Ghosh, Eur. Polym. J., 17, 545, 817 (1981).

8. S. G. Cohen, G. A. Davis, and W. D. K. Clark, J. Am. Chem. Soc., 94, 869 (1972).

9. R. F. Bartholomew and R. S. Davidson, J. Chem. Soc. (C), 2342, 2347, (1971).

10. S. Tazuke, "Developments in Polymer Photo- 
chemistry-3," N. S. Allen, Ed., Applied Science Publishers, London, 1982, p 62.

11. C. David, W. Demarteau, and G. Geuskens, Polymer, 10, 21 (1969); G. Oster, G. K. Oster, and H. Moroson, J. Polym. Sci., 34, 671 (1959).

12. G. Sánchez, G. Weill, and R. Knoesel, Makromol. Chem., 176, 131 (1978).

13. G. J. Smets, Polym. J., 17, 135 (1985); G. J. Smets, S. N. E. Hamouly, and T. J. Oh, Pure Appl. Chem., 56, 439 (1984).

14. K. Tsubakiyama, M. Yamamoto, and Y. Nishijima, J. Polym. Sci., A, Polym. Chem., 26, 1231 (1988).

15. C. S. Marvel, C. G. Overberger, R. E. Allen, and J. H. Saunders, J. Am. Chem. Soc., 68, 736 (1946).

16. A. Tsuchida, M. Yamamoto, and Y. Nishijima, $J$. Phys. Chem., 88, 5062 (1984).

17. A. Charlesby and S. H. Pinner, Proc. Ro. Soc., London, Ser. A, 249, 367 (1959); H. H. G. Jellinek, J. Appl. Polym. Sci, Appl. Polym. Symp., No. 4, 41
(1967).

18. J. Chilton, L. Giring, and C. Steel, J. Am. Chem. Soc., 98, 1865 (1976); C. Brauchle, D. M. Burland, and G. C. Bjorklund, J. Phys. Chem., 85, 123 (1981); K. Horie, H. Ando, and I. Mita, Macromolecules, 20, 54 (1987)

19. K. Tsubakiyama, S. Ito, M. Yamamoto, and Y. Nishijima, Polym. Prepr. Jpn., 33, 289, 1475 (1984).

20. H. A. Hammond, J. C. Doty, T. M. Laakso, and J. L. R. Williams, Macromolecules, 6, 711 (1970).

21. R. Bensasson and E. J. Land, Trans. Faraday Soc., 67, 1904 (1971); D. I. Schuster and M. D. Goldstein, Mol. Photochem., 7, 209 (1976).

22. K. Tsubakiyama, A. Tsuchida, M. Yamamoto, and Y. Nishijima, Preprints, The 51st Annual Meeting of the Chemical Society of Japan, Kanazawa, 1985, II, $\mathrm{p} 885$.

23. C. G. Shaefer and K. S. Peters, J. Am. Chem. Soc., 102, 7566 (1980). 